\title{
From Control of the Electron Beam to Control of Single Atoms
}

Andrew R. Lupini ${ }^{1,2^{*}}$, Bethany M. Hudak ${ }^{1}$, Stephen Jesse ${ }^{1,2}$, Jiaming Song ${ }^{3, \#}$, Ondrej Dyck ${ }^{1,2}$, Paul C. Snijders $^{3}$ and Sergei V. Kalinin ${ }^{1,2}$

1. The Center for Nanophase Materials Sciences, Oak Ridge National Laboratory, Oak Ridge, TN, USA.

2. The Institute for Functional Imaging of Materials, Oak Ridge National Laboratory, Oak Ridge, TN, USA.

3. Materials Science and Technology Division, Oak Ridge National Laboratory, Oak Ridge, TN, USA.

\#. Present address: School of Physics, Northwest University, Xi'an, China.

* Corresponding author: arl1000@ornl.gov

Control of dopant atoms and defects in materials underpins much of modern electronics and holds great promise for future applications and 'quantum' technologies. However, from the early days of the electron microscope its resolution was limited by the aberrations of the electron lenses to just beyond the level where such features can be directly visualized. Thus the development of modern aberration-correctors, which pushed the feats of imaging individual atomic columns or even single atoms to a more routine level, has propelled electron microscopy to the forefront of many areas of materials science.

One of the outcomes of reduced aberrations is alleviation of the electron wavelength resolution limit, resulting in the ability to obtain atomic-resolution images at a wider range of accelerating voltages. This allows us to select the operating energy of the electron microscope, and in doing so vary the amount of energy that is transferred to the sample. For example, graphene and similar 2D materials are often best imaged at voltages around $60 \mathrm{kV} \mathrm{[1]}$, whereas imaging at higher energies can quickly modify the area of interest. However, operating the microscope at a range of voltages presents a range of challenges, varying from the technical difficulties of aligning the aberration-corrector [2] to minimizing thermal drift. Another of the key uncertainties in the precise interpretation of scanned microscope images is knowing exactly where the probe is on the sample. This difficulty arises because the beam will deviate from the desired position due to limitations in the control electronics or drift in the sample position. A closely linked strategy to limit undesired sample damage is therefore to precisely control the beam. The electron beam can be scanned in variable patterns, such as spirals centered on the area of interest [3]. This control can be used to more accurately determine the probe position, to characterize the response of the scan system and ultimately to guide the beam to only the desired coordinates.

Recent work has begun to open a new era in electron microscopy, where instead of being used only as an imaging or spectroscopic system, the atomic-sized electron beam in a scanning transmission electron microscope (STEM) can be used as a tool to manipulate matter at the nanoscale, with examples including fabricating crystalline oxides at atomic-plane resolution [4] and sculpting the technologically ubiquitous silicon [5]. For 2D materials, this control has been extended to single atoms, as demonstrated by moving single silicon atoms in graphene [6,7]. In 3D crystalline materials, recent developments have allowed the imaging of single atom diffusion [8] and control of single dopant atoms [9] (see Figure 1). Combining variable operating voltage electron microscopy with position control therefore represents a promising strategy to either reduce undesired damage or to engineer a new atom-by-atom fabrication technique [10].

References:

[1] OL Krivanek et al., Nature 464 (2010). 
[2] N Dellby et al., J. Electron Microsc. 50 (2001), p. 177.

[3] X Sang et al., Scientific Reports 7 (2017).

[4] S Jesse et al., Small 11 (2015), p. 5895.

[5] S Jesse et al., Nanotechnology 29 (2019), p. 255303.

[6] T Susi et al., Ultramicroscopy 180 (2017), p. 163.

[7] O Dyck et al., Applied Physics Letters 111 (2017), p. 113104.

[8] R. Ishikawa et al., Phys Rev Lett 113 (15) (2014).

[9] BM Hudak et al., ACS Nano 12 (2018), p. 5873.

[10] Work supported by the U.S. Department of Energy, Office of Science, Basic Energy Sciences, Division of Materials Science and Engineering, and by the Laboratory Directed Research and Development Program of Oak Ridge National Laboratory, managed by UT-Battelle, LLC for the U.S. Department of Energy, and was performed at the Oak Ridge National Laboratory's Center for Nanophase Materials Sciences (CNMS), a U.S. Department of Energy Office of Science User Facility.
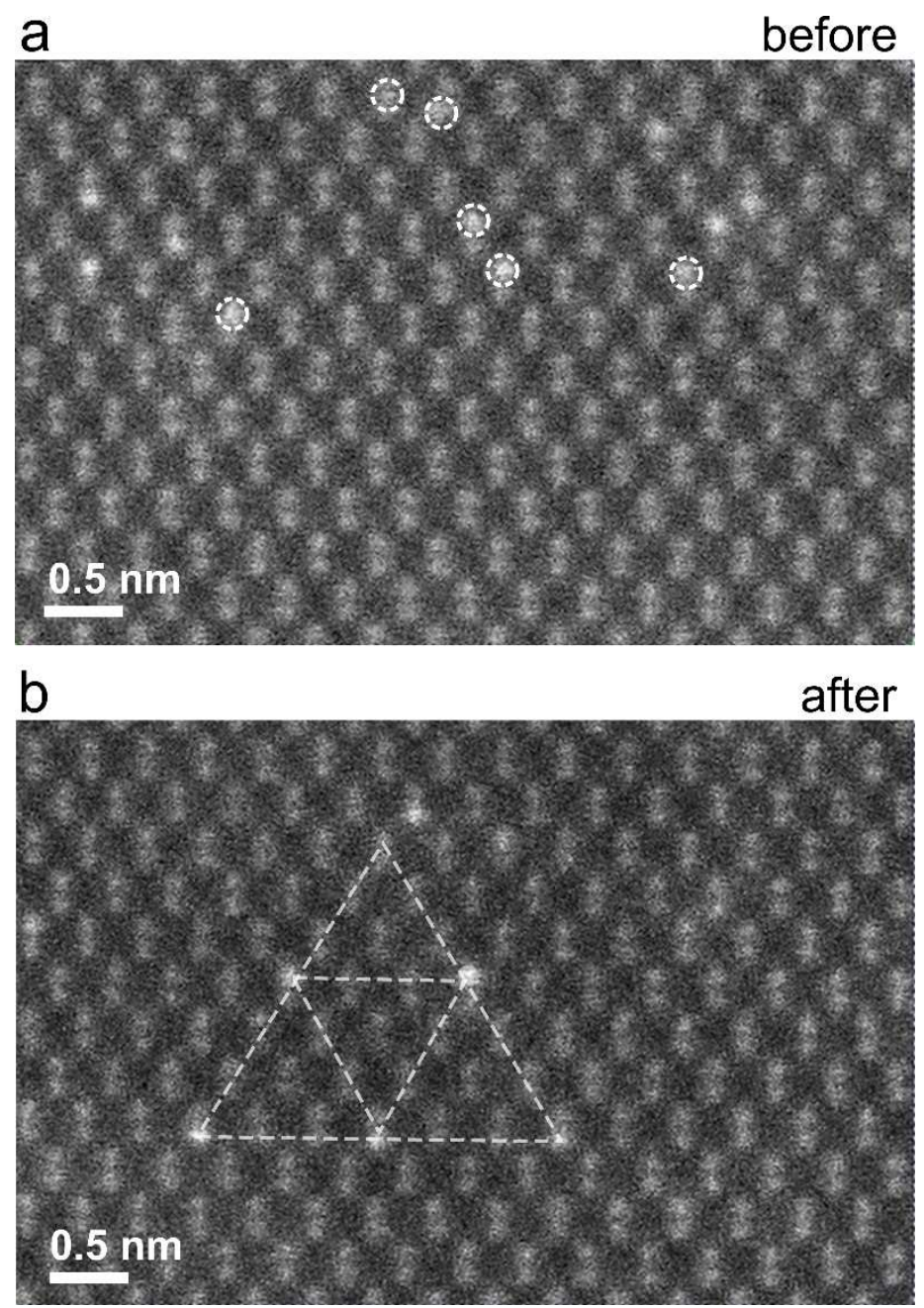

Figure 1. High-angle annular dark field (HAADF) images showing single dopant Bi atoms in Si[110]. A $160 \mathrm{kV}$ electron beam was used to guide the atoms towards the desired position. Images adapted from reference [9]. 